\title{
Risk Management: An Epidemiologic Approach
}

The risk of adverse outcomes which accompanies admission to hospitals has often been overshadowed by the promise and hope of restoration of health. In recent decades, however, a litigation-conscious society, concern for quality care, and increasing bureaucratic intervention into health care have focused attention on hazards of hospitalization. The list of nosocomial complications is multifarious and includes adverse drug reactions, complications of surgery, complications of diagnostic procedures, transfusion reactions, infections, falls, burns, and irradia'tion injuries. ${ }^{1,2}$ Consequences of these nosocomial complications range from minor, temporary injuries to chronic, irreversible disability and occasionally death. Aside from the personal consequences of the injury, there is an added burden of increased cost to the hospital industry and to society. It is estimated that among 23 different industrial groups, the "hospital" industry was second only to "construction" in the industry-wide cost of injury as a percent of revenues in $1980 .^{3}$

Among the risks of hospitalization, only nosocomial infections have been subjected to systematic scrutiny and analysis. With well-defined epidemiological tools, risks have been identified, interventions implemented, and cost-effectiveness confirmed. ${ }^{4}$ Other hazards of hospitalization received little attention until increased public 'awareness of nosocomial injuries brought pressure on the hospital industry, primarily through the legal system. A

From the Division of Clinical Epidemiology, Departments of Internal Medicine and Preventive Medicine, University of Iowa, lowa City, Lowa.

Address reprint requests to R.M. Massanari, MD, Associate Hospital Epidemiologist, The University of Iowa Hospitals and Clinics, Program of Epidemiology, Iowa City, IA 52242. plethora of successful malpractice suits has resulted in astronomical increases in the cost of liability insurance premiums. ${ }^{5}$ To counter these rising costs, risk management programs have been proposed to identify and manage adverse, nosocomial events. ${ }^{6}$ In some states where the cost of malpractice is excessive, risk management has been mandated through legislation. ${ }^{7,8}$ In contrast to infection control programs which have developed on sound epidemiological principles, risk management utilizes the empiric case analysis modus operandi. It is the purpose of this editorial to subject current risk management to a critique and to propose a more sound epidemiological approach to the identification, analysis and control of hospital hazards.

\section{RISK MANAGEMENT}

The primary impetus for risk management programs is reduction in costs associated with malpractice suits. This objective is epitomized in the following statement: "An ounce of malpractice prevention is worth a ton of money." While humanitarian objectives are espoused, there is, nevertheless, a basic philosophical underpinning different from the one of advocates for the prevention of untoward events. It is not the objective of this review to debate the philosophical and ethical issues, but rather to focus on the potential for inefficiency and misleading conclusions which may emerge from an empiric approach to risk assessment.

Craddick developed a methodology for identification and management of incidents which may result in litigation. ${ }^{6}$ The tool has been widely accepted with minor modifications and is being implemented in hospitals across the country. ${ }^{9-11}$ The methods for risk analysis may 
be summarized briefly. Using criteria proposed by Craddick, ${ }^{6}$ one can screen all patient records for incidents or untoward events which might result in malpractice suits. The data which are collected include demographic information regarding the patient and the event. In addition, lengthy narrative summaries are required for empiric case analysis. The summaries are prioritized so that more serious problems are referred to designated reviewers, usually nurses, physicians and/or lawyers. ${ }^{12}$ The reviewer analyzes each case using implicit criteria to develop a course of management to mitigate the risk of litigation. From the analyses, reviewers may propose interventions to prevent recurrence of the nosocomial complication. Monitoring trends of events is encouraged, but the program offers no concrete methods for achieving the objective. Re-evaluation of interventions to assess efficacy is not discussed in procedure manuals.

To recapitulate, the primary objective of the program is to reduce the risk of malpractice which may result from nosocomial complications. To presume that the program will achieve that objective, one should demand critical evaluation. The following discussion will review some of the limitations of current risk management programs.

\section{Surveillance}

Insistence on $100 \%$ surveillance ignores the rationale underlying sample techniques. ${ }^{13}$ Hospital epidemiologists involved with infection control advocate focused surveillance which concentrates limited resources in areas of high risk. ${ }^{4,14}$ Concurrent surveillance of all patients for adverse occurrences is an inefficient use of resources.

The criteria used for surveillance have not been characterized. A review of the literature suggests that there have been no efforts to evaluate the sensitivity and specificity of the screening tool. ${ }^{15}$

\section{Analysis of Data}

Since the focus of the analysis is management of the incident after the fact (in order to reduce the risk of liability), there is no systematic analysis of predisposing factors. Risk factors are identified from empiric observations of one or two reviewers. From observations of only one or two patients, elaborate, costly interventions have been developed and implemented throughout an institution. ${ }^{16}$ A superficial analysis of predisposing factors fails to consider the often multifactorial contributions to adverse outcomes and, indeed, may overlook entirely a critical determinant when the factor in question is a confounder.

\section{Evaluation of Interventions}

Follow-up of interventions after implementation is essential to assure that the control was effective. This step in risk assessment is not advocated in risk management programs. Based upon the type of data collected for analysis, it would be difficult to perform such an analysis.

\section{Documentation of Efficacy}

There is no documentation that risk management methodologies will reduce costs associated with adverse events. ${ }^{15}$ Since the principal objective of the program is reduction in malpractice suits and since suits are infrequent consequences of the many adverse events which occur in hospitals, documentation of efficacy will be difficult.

In summary, insistence on $100 \%$ screening and development of educational exercises and interventions based on analyses of single incidents foster inefficiency. If the primary objective of risk management is cost reduction, a critical analysis of the cost-effectiveness of the program should be imperative. Published documentation of the efficacy of risk management programs for reducing costs associated with hospital hazards is not available and will be difficult to document because data retrieval is inadequate. It is, indeed, conceivable that the cost of operating an inefficient program may outweigh any potential benefits in a community where litigation is infrequent. $^{17}$

\section{RISK DEFINED AS PROBABILITIES}

Up to this point in the discussion, risk has not been defined. It is in part intentional, since the term is illdefined in risk management programs. Implied is management of the "risk" of litigation following untoward events resulting from hospitalization. Although the number of such events may be reported and analyzed with " regard to site of occurrence and relative to patient demographics, quantification of the risk of an event is almost never reported. ${ }^{18}$ Indeed, collection of data as outlined in ' most risk management programs will not allow quantification of risk.

Application of epidemiological tools to risk analysis in the hospital requires a definition which can be quantified. While it is possible to determine the risk of litigation following untoward events, it occurs irregularly and so infrequently that analysis and interpretation of findings would be limited. Litigation, however, is a consequence of events such as errors of omission or commission. Such events occur with regularity in hospitals. ${ }^{1}$ By focusing the analysis on events which predispose to litigation, one can design interventions to prevent occurrence and thereby improve the quality of medical care. It follows that the likelihood of litigation should decrease as the risk of untoward events decreases. For this discussion, risk will be defined in terms of the frequency or probability of occurrence of an untoward event and in terms of some measure of the severity of the consequences of the event. ${ }^{19}$

$$
\text { Risk }=\text { Probability } \times \text { Severity }
$$

Both components of the definition are important in establishing priorities in risk control programs. If the probability of an event is infrequent and has minimal consequences for the patient, it should demand a low level priority. On the other hand, if the event is infrequent but is accompanied by grave consequences, ie, transfusion reaction, a much higher priority is imperative.

The estimation of probabilities of events requires two sources of data. First, the number of events must be determined. Identification and counting of events in order to determine probabilities will require an accurate estimate of all events which incur risk of adverse consequences. Note that to obtain an accurate estimate of events will not require surveillance of all patients provided a proper sample of the population is selected. ${ }^{13}$ Valid 
TABLE

PROBABILITIES OF NOSOCOMIAL RENAL FAILURE IN HOSPITAL $X$

A HYPOTHETICAL PROBLEM

\begin{tabular}{lcccccc}
\hline Renal & \multicolumn{2}{c}{ Drug A } & \multicolumn{2}{c}{ Drug B } & \multicolumn{2}{c}{ Drugs } \\
Failure & Hospital & Literature & Hospital X & Literature* & A \& B & IVP \\
\hline Yes & 5 & 10 & 5 & - & 5 & 3 \\
No & 245 & 990 & 95 & - & 5 & 177 \\
Total & 250 & 1000 & 100 & - & 10 & 180 \\
Probability & 0.02 & 0.01 & 0.05 & - & 0.5 & 0.016
\end{tabular}

*No Information.

retrieval of events will depend on the sensitivity and specificity of the criteria used to define the event. A discussion of this facet of risk analysis is beyond the scope of the current review. Second, one must identify and count the number of patients who may be at risk for the event during a given period of time. For example, to determine the probability of postoperative hemorrhages for a 1month period, one will need to determine the number of postoperative hemorrhages occurring during the same month and divide by the number of patients at risk, ie, the number of patients who underwent surgery during the same time period. This probability may be defined as the cumulative incidence of the event (hemorrhage).

The selection of a denominator is critically important in developing a model for analysis. ${ }^{19}$ For example, when investigating complications of intravenous therapy, it may be more useful to determine the number of complications (events) per number of days IVs are in place than to determine the number of complications per patient(s) with IVs. Expressing the frequency of events per time of exposure is defined as incidence density.

The third source of data necessary to determine risk is an estimation of the severity of the injury resulting from the event. Scales for determining the severity of the injury are included in risk management programs ${ }^{9}$ and should be applicable to most epidemiological investigations.

An illustration of the relative utility of empiric case analysis vis-á-vis epidemiological analysis may be useful.

Risk management criteria insist that any unexplained organ system failure which occurs during hospitalization be screened. Renal failure occurring after admission to a hospital is an example of a potentially preventable, severe nosocomial complication. Suppose that during May 1986, in Hospital X, six patients experienced unexplained acute renal failure. Five of six patients were receiving drugs $\mathrm{A}$ and $\mathrm{B}$. The sixth patient had diabetes mellitus and had undergone an IVP. Two of five patients receiving drugs $A$ and $B$ had also had IVPs. Case analysis would make it difficult to determine which of the several factors cited contributed to acute renal failure (eg, Drugs A, B, diabetes mellitus, and/or the intravenous pyelogram). If perusal of the literature indicated that drug $\mathrm{A}$ had been associated with occasional renal failure, the reviewer might erroneously conclude that drug $\mathrm{A}$ was the principal cause and intervene to prevent further events by eliminating the agent from the formulary.

Using epidemiological tools to evaluate the problem, one would attempt to determine the rate or probability of renal failure associated with the several factors described. Several probabilities for renal failure in Hospital $\mathrm{X}$ are calculated in the Table. Note that while the probability of renal failure for patients taking drug $A$ exceeds the expected (endemic) rate described in the literature, it does not approach the probability associated with taking drugs $A$ and $B$ simultaneously. Therefore, there appears to be a heretofore unrecognized interaction between drugs $A$ and $B$.

It should be noted that the high probability of developing renal failure when taking the two drugs simultaneously does not necessarily indicate a cause and effect relationship. However, more rational immediate measures to prevent the recurrence of the untoward event can now be instituted in Hospital $\mathrm{X}$ as well as elsewhere in the medical community. Instead of denying the benefits of a potentially useful agent, drug $A$, one need only restrict the simultaneous use of drugs $A$ and $B$ to a few patients. Finally, having established the rate of renal failure during the "epidemic," trends in frequency of renal failure may be followed over time to assess the efficacy of the proposed intervention. If the hypothesis that combined use of drugs A and B causes renal failure is correct, the probability of renal failure should diminish after implementing the intervention.

This hypothetical problem illustrates how case review by one or two observers using implicit criteria may lead to fallacious conclusions and inefficient management of the problem. On the other hand, quantification of risk in terms of probabilities allows for a more critical assessment of the data and can be subjected to the critique of several individuals.

\section{UTILIZATION OF RATES AND PROBABILITIES IN RISK ANALYSIS}

In contrast to the empirical approach used in case analysis, defining risk as probability permits a systematic analysis of problems and allows one to examine and test the efficacy of the solution.

\section{Comparing Rates or Probabilities}

Implied in the problem of renal failure described above was the notion that for any risk factor there is a probability of adverse outcome even under optimal circumstances. This rate may be called the endemic rate and can be determined for any institution and, if numbers are sufficient, for any known risk factor within that institution. An 
increase in the rate or probability of renal failure (event) would signal a potential problem (epidemic) which would mandate a more thorough analysis. ${ }^{20}$ Monitoring rates rather than individual events or cases will provide a more efficient method of concurrent surveillance for many untoward events.

Simply identifying a rise or fall in the rate of an event is not sufficient to conclude that an epidemic has ensued or resolved. Rates vary over time simply by chance. To conclude that a change in rate is significant, ie, the change is not simply due to chance phenomenon, one should apply a statistical test. Since the probability of adverse events has already been quantitated, it is relatively easy to evaluate the significance of the change by calculating a chi-square statistic and confidence intervals. ${ }^{21}$ Conclusions based on this type of epidemiologic analysis are considerably more reliable than those based on empiricism. Rates may also be compared over time for trend analysis or compared among institutions to assess the impact of intervention programs and quality of care. ${ }^{22}$

In some instances, one will be faced with adverse outcomes for which the risk factor is still uncertain. Applying epidemiological methods, the probability of exposure to a presumed risk factor among the "cases" may be compared with the probability of exposure to the same factor among non-cases or "controls." The case-control method provides an efficient tool for analysis of risk under these circumstances. ${ }^{23}$

\section{Analysis of Multiple Factors}

Nosocomial complications often result from a series of exposures or events. Multivariate analysis techniques applied to nosocomial infections have provided insight into the complexity of factors which predispose patients to hospital acquired infections. ${ }^{24-26}$ These techniques are useful in identifying multiple factors which must be addressed when intervening in the chain of events leading to undesirable outcomes. With one exception, these techniques have not been applied to analysis of risk other than infection. ${ }^{15}$ Estimation of probabilities of outcomes (dependent variables) and probabilities of exposures or untoward events (independent variables) makes it possible to develop models for analysis of risk. This sophisticated form of risk assessment is inconceivable with a caseby-case analysis.

\section{Risk-Benefit Analysis}

For longer term health care planning, decisions are ideally based on costs and benefits associated with the risk of a particular procedure or event. Under circumstances in which risks are high, or alternatively low, empiric observations are often adequate for making effective decisions. To return to the example of the interaction of drugs $A$ and $B$, the risk of combining the two agents far exceeded any minimal benefit that might accrue. Many decisions regarding risk are not so obvious, however, and require more sophisticated analyses which are not possible with simple empiric analysis. These analyses require quantification of risk as described above. ${ }^{19}$

Alternative techniques may be used to predict potential benefits of risk control. From comparisons of rates of exposure among cases and controls (odds ratios), attribut- able risk may be calculated. ${ }^{27}$ Attributable risk provides an estimate of benefit which will accrue to the exposed population of patients by controlling a known event or risk factor. These estimates are only possible when sufficient data are available to quantify risks, however.

Control of hazards of hospitalization and reduction of untoward nosocomial complications is in keeping with quality medical care. Methods for achieving these ends deserve careful scrutiny, however. Risk management programs which utilize implicit criteria and empirical observations of individual cases have limited utility in risk analysis. Presently, there is little to document the efficacy of these programs in achieving their stated objective. On the contrary, there is now well documented evidence to support the use of standard epidemiological techniques in the control of one specific hazard of hospitalization: nosocomial infections. It seems reasonable, therefore, to extend the application of these "tried and true" techniques to the analysis and control of other risks associated with hospitalization.

\section{REFERENCES}

1. Schimmel EM: The hazards of hospitalization. Ann Intern Med 1964; 60(1):100-110.

2. Lee RG: Health and safety in hospitals. Med Sci Law 1979; 19(2):89-103.

3. Brown BM: How financial managers can reduce their risk and insurancerelated costs. Healthcare Financial Management, March 1984, pp 32-35.

4. Haley RW, Culver DH, White JW, et al: The efficacy of infection surveillance and control programs in preventing nosocomial infections in US hospitals. Am J Epidemiol 1985; 121(2):182-205.

5. Munch P: Causes of the Medical Malpractice Insurance "Crisis": Risks and Regulations. Santa Monica, CA, The Rand Corporation, 1976.

6. Craddick JW: The medical management analysis system. $Q R B$ 1979; 5(4):2-8.

7. An Act Relating to Medical Malpractice and Medical Liability Insurance Amending s627.352(5). Florida Statutes $\$ 395.18$, c. $76-260$, no. 586, 1976.

8. Plan of Operation. Review, Approval, Amendment, Reports. Michigan Statutes, $\$ 500.2507$, P.A. 1975 , No. $43,1975$.

9. St. Paul Fire and Marine Insurance Company: The Occurrence Screening System. St. Paul, MN, St. Paul Fire and Marine Insurance Company, 1980.

10. Blake P: A complete guide. Nurs Management 1984; 15(11):37-41.

11. Bloom A: Risk management in medical groups. Med Group Management, March/April 1983, pp 10-12.

12. Freilich H: Rapid incident identification, systematic follow-up key to preventive risk managing. Hospitals 1982; 56(16):53-54.

13. Chavigny KH, Fischer J: Nosocomial infection in a high risk cohort: An illustration of a sampling method. Infect Control 1983; 4(1):19-24.

14. Wenzel RP, Osterman CA, Hunting KJ: Hospital-acquired infections. 11. Infection rates by site, service and common procedures in a university hospital. Am J Epidemiol 1976; 104:645-651.

15. Elnicki RA, Schmitt JP: Contribution of patient and hospital characteristics to adverse patient incidents. Health Serv Res 1980; 15:397-414.

16. Oulton R: Use of incident report data in a system-wide quality assurance/risk management program. $Q R B 1981 ; 7(6): 2-7$.

17. Stearns G, Fox LA: Assessing quality assurance and risk management activities: A profile analysis. $Q R B$ 1979; $5(10): 26-29$.

18. Latessa P, Long G, McCracken SB: Incident data compiled and compared. Hospitals $1979 ; 53(22): 54-58$.

19. Wilson R, Crouch E: Risk/Benefit Analysis. Cambridge, MA, Ballinger Publishing Company, 1982; pp 9-10, 61-62, 71-72, 93-94.

20. Buehler JW: Changes in mortality rate may signal increased risk. Hosp Risk Management, August 1983, pp 101-103.

21. Veney JE, Kaiser DL: Contingency tables for tests of differences between groups. Infect Control 1980; l(6):417-423.

22. Williams RL: Measuring the effectiveness of perinatal medical care. Med Care $1979 ; 17(2): 95-111$.

23. Kantor RJ, Carson LA, Graham DR, et al: Outbreak of pyrogenic reactions at a dialysis center. $A m J M e d$ 1983; 74:449-456

24. Freeman J, McGowan JE: Risk factors for nosocomial infection. J Infect Dis $1978 ; 138(6): 811-819$.

25. Hooton TM, Haley RW, Culver DH, et al: The joint associations of multiple risk factors with the occurrence of nosocomial infection. Am J Med 1981; 70:960-970.

26. Haley RW, Culver DH, Morgan WM, et al: Identifying patients at high risk of surgical wound infection: A simple multivariate index of patient susceptibility and wound contamination. Am J Epidemiol 1985; 121(2):206-215.

27. Miettinen OS: Proportion of disease caused or prevented by a given exposure, trait or intervention. Am J Epidemiol 1974; $99(5): 325-332$. 